\title{
A FRAMEWORK FOR ENHANCING AND GUARDING THE RELEVANCE AND QUALITY OF SCIENCE: THE CASE OF THE CGIAR ${ }^{1}$
}

\author{
By A. H. KASSAM*+, H. M. GREGERSEN+, E. FERERES+, E. Q. JAVIER+, \\ R. R. HARWOOD + and A. de JANVRY+ \\ +CGIAR interim Science Council, Food and Agriculture Organization (FAO), Rome, Italy
}

\section{SUMMARY}

The CGIAR Technical Advisory Committee (TAC) is being transformed into a Science Council. This paper is about the role of the CGIAR Science Council in enhancing and guarding the relevance and quality of science in the CGIAR. In carrying out this role, the Science Council must act in a strategic advisory role, basing its advice on: planning and strategy development in the context of CGIAR goals; internal self-assessments and independent external monitoring and evaluation; and on impact assessments. The paper elaborates these three main functions of the Science Council to facilitate the transformation of TAC into a full Science Council.

\section{INTRODUCTION}

The basic role of the Science Council (SC) of the CGIAR ${ }^{2}$ is to enhance and guard the relevance and quality of science in the CGIAR. In carrying out this role, the SC acts in a strategic advisory role, basing its advice on assessments, planning and evaluation functions. In this context, relevance can be viewed as researching the "right things" to address the System's goals, and quality as researching the "right things well"".

For science to be relevant in the context of the CGIAR, it should produce solutions that will have measurable, significant impact in terms of the goals of the System. Thus, the relevance of the identified research priorities and strategies to the CGIAR goals and mission, and the nature of the planning and consultation process, including ex-ante analyses of need and potential impact to formulate the priorities and strategies, are important elements in defining relevance. The CGIAR vision and strategy (TAC, 2000) provides a comprehensive framework for judging relevance Planning for relevance is increasingly seen as an inclusive

\footnotetext{
* Corresponding author: Email: amir.kassam@fao.org

${ }^{1}$ Based on a paper prepared by the authors to facilitate the transformation of the CGIAR Technical Advisory Committee (TAC) into Science Council (SC). An earlier draft was discussed at the iSC/TAC 82 meeting in April 2002, and revised draft at iSC/TAC 83 meeting in August 2002.

${ }^{2}$ Created in 1971, the Consultative Group on International Agricultural Research (CGIAR) is an association of more than 50 public and private members that support a system of 16 international agricultural research centres. Membership included developing and industrialized countries, private foundations and regional and international organizations. World Bank, FAO, UNDP and IFAD serve as Co-sponsors.

${ }^{3}$ This definition of relevance and quality does not exclude the treatment of relevance as a dimension of quality, and vice versa.
} 
process requiring greater interface with the stakeholders of CGIAR research, not only donors, national agricultural research systems (NARS) and NGOs, but also organized groups of poor people (producer organizations, grassroots organizations). Demand for CGIAR research services should really originate in part from these stakeholder groups, particularly as they must also be involved in facilitating the effective implementation of research. This bottom up process of planning for relevance and coordinated implementation has been stressed in the CGIAR vision and strategy.

Quality of science concerns four components, namely: inputs, processes, and outputs and outcomes of science. It has to do with the correct formulation of hypotheses, and with the appropriateness of scientific inputs, research methodologies and processes, and research outputs and outcomes. At the input end, the research "tools" (whether analytical, instrumental etc.) and scientific and disciplinary expertise being applied to do the research work are important quality parameters. Are tools and disciplines appropriate to the task being employed? Are new methods and techniques and new science being judiciously introduced? Thus quality of research inputs has to do with the appropriateness of the tools, methods and disciplines employed. As for quality of research process, what is the evidence that the research work being done is well executed and managed, including how effectively are the research milestones being achieved? At the output end, quality of research products has to do with scientific achievements and intermediate and ultimate outcome or impact. Thus, scientific quality deals with the efficient use of state of the art knowledge, research methods and protocols.

Within these definitions of quality and relevance, the paper serves as a starting point for the development of specific criteria, procedures and guidelines for carrying out the responsibilities envisioned for the SC.

Progress in the CGIAR involves a dynamic process, an iterative one of successive approximations as the System adapts to the changing agricultural science and development environments and needs in which it finds itself. This overall process of operation of CGIAR is laid out in Table 1, along with the context in which it takes place, and the role envisioned for the SC in the context of the activities undertaken by the other stakeholders in the CGIAR. The sections that follow consider each of the stages in the process outlined.

Thus, Section 2 looks at the SC's role in research planning and strategy development, given the goals and boundaries. Section 3 goes on to suggest the SC role in the organization and choice of research programme modalities, while Section 4 suggests the SC role during implementation of research. Section 5 then discusses the SC role in assessing the impacts of the CGIAR, through its research and its capacity strengthening activities. Section 6 suggests the potential role of the SC in making sure that past and present experience feeds back into planning and strategy development as the System moves through its iterative process of changes and adaptation to the changing environment. 


\section{RESEARCH PLANNING AND STRATEGY DEVELOPMENT}

A first and fundamental task of the CGIAR is to develop the overall plans and strategies for implementing its research and related activities aimed at achieving its goals.

\section{Scientific and Development Context}

The main business of the CGIAR is the application of science for development leading to a food secure world for all. The CGIAR System's overarching mission is to achieve sustainable food security and reduce poverty in developing countries through scientific research and research-related activities in the fields of agriculture, livestock, fisheries, policy and natural resource management. Its overarching goal is to reduce poverty, hunger and malnutrition by sustainably increasing the productivity of resources in agriculture, forestry and fisheries. In a dynamic and political world, these goals need to be revisited on a regular basis to make sure that they are in tune with the needs regarded as priorities by the global fraternity of nations and institutions that support agricultural and related research for development and that benefit from it.

The new CGIAR vision and strategy calls for, inter alia, an increased emphasis on pro-poor relevant research, on regional and bottom up orientation, on bringing new science to bear on the often difficult-to-address causes of poverty and food insecurity, on the reorienting of the CGIAR towards undertaking critical Challenge Programmes. With these fundamental changes, the CGIAR is entering a new phase in its development. The new phase requires a much stronger concern for higher standards of scientific relevance and quality, for keeping pace with state of the art in world research, and for being relevant, selective and targeted.

Given that the CGIAR accounts for a very small part (less than 5 percent) of agricultural research in the developing regions, it focuses mainly on strategic and applied research of an international public goods nature, thus respecting the notion of division of labour between itself and its NARS partners ${ }^{4}$. As the ultimate responsibility for the adaptation and dissemination of improved knowledge and technologies rests with NARS and extension and development agencies, the CGIAR plays a catalytic role in the delivery of research products to the beneficiaries with whom it conducts participatory research and technology development work. It is for this reason that the capacity strengthening (research-related) role of the CGIAR is considered to be important and necessary.

\section{Role of the Science Council}

Relevant activities and strategies of operation for the CGIAR have to be chosen in this context of the existing and evolving global science and development environments in which CGIAR research exists. Further, priorities for CGIAR activity have to be planned and developed considering what others are doing and can do more effectively and efficiently than the CGIAR. The SC can maximize its support to the Executive Council (ExCo) of the CGIAR in planning and making the necessary choices of strategy by:

\footnotetext{
4 NARS, or National Agricultural Research Systems is an inclusive term that includes entities engaged in agricultural research and related activities in the public, private and civil society sectors. Thus, they include universities, national public agricultural research institutes, NGO organizations, private sector and farmer groups that are involved in improving agriculture, broadly defined, through research.
} 
- monitoring changes in the global science and development environments and appropriately engaging in strategic and operational planning to suggest the appropriate role for the CGIAR in the context of these changes;

- monitoring the research and capacity strengthening activities conducted by others, and leveraging the comparative advantage of the CGIAR in given areas of activity, particularly through cooperation with NARS; and

- suggesting, in the context of these assessments of the global science and development environments, how the CGIAR strategically should proceed to carve out its niche and produce relevant, high quality, state of the art research to address the problems in which it has a comparative advantage.

Using the results of these SC assessments, the Centres and the ExCo, working with outside partners, can decide on an appropriate set of plans and strategies for organizing and implementing CGIAR research. It should be emphasized that the content of the CGIARsupported research and related activities implied by the resulting plans need to be derived in a consultative, sequential and iterative manner.

More specifically, this role in strategic planning is a fundamental anchor role of the SC in the CGIAR System as described in the responsibilities assigned to the SC by the CGIAR. The SC should keep a watching brief on the global and regional external research and development environments and provides ongoing, independent and objective advice to the Group and its Centres on the context and significant trends that should affect priority setting and strategy development processes in the CGIAR. An important role for the SC here is also to keep the CGIAR informed of stakeholders' evolving needs and demands for new research and collaboration. This can be done through special studies (poverty analyses, regional analyses, etc.) but also through interactions with stakeholders (voices of the poor, interactions with regional organizations, etc.). This provides a basis for formulating the strategic planning context at the CGIAR System level.

To help focus on specific challenges or the potentials for new scientific applications, the SC would:

- interact with a wide range of experts and peers from the global science and development communities, to understand better the relevant scientific and development contexts;

- commission or mount itself ex-ante and ex-post strategic studies of relevant crossCentre themes and activities (also of interest to CGIAR Members and the Centres) to help inform the formulation of plans and strategies;

- undertake periodic stripe evaluations of cross-Centre themes and topics to monitor science relevance and quality and formulate recommendations for future directions in research;

- organize and implement studies of regional research commitments of CGIAR in the context of Plank 4 of the CGIAR vision and strategy; and 
- commission ex-post impact assessments of CGIAR research;

- keep aware of the factors affecting the comparative advantage of the CGIAR.

The results of all of these activities would feed into periodic reassessments and ongoing planning of the overall research agenda of the CGIAR.

Ex-ante strategic evaluations and perspective studies are undertaken to help identify, prioritize, plan and formulate science policy, priorities and strategies needed to most effectively and efficiently implement the CGIAR vision and goals., Such evaluations underpin the scientific advice and the debates that lead to the formulation and agreement on a strategic framework for reaching consensus on priorities, and for preparing the planning context and guidance for the System as well as Centre level strategic planning. These strategic evaluations and studies have been handled through stripe and inter-centre evaluations (e.g. farming systems, training, rice), strategic studies (e.g. plant genetic resources, soil and water, marginal lands, roots and tubers, post harvest), and regional studies (e.g. West Africa, Latin America). The SC would continue to provide such strategic analysis. The iSC is revising its existing basic document on strategic planning, in consultation with $\mathrm{CBC}$ and $\mathrm{CDC}$ and other stakeholders, and in the light of the new responsibilities of the SC. This will be available to the $\mathrm{SC}$ as it formulates its approach to meeting its assigned responsibilities.

With the addition of CPs to the array of research and capacity strengthening mechanisms, and with the complexity and time lines introduced by these new mega programmes, the role of strategic planning and evaluation of CGIAR future directions becomes all the more important. Since the new CPs represent major commitments of resources over longer periods of time and across Centres (i.e., at the System level), it is even more essential than before that the SC devote adequate time and effort to understand and prioritize relevant themes and the quality of the science proposed. TAC in the past has periodically prepared a System level "priorities and strategy" document for discussion and eventual approval by the Group.

\section{ORGANIZATION OF RESEARCH: PROGRAMME STRUCTURE, ACCOUNTING AND GOVERNANCE ${ }^{5}$}

The totality of the CGIAR's research and research-related activities may be summarized into five major outputs of the System log-frame format, namely: germplasm management, germplasm improvement, sustainable production systems through INRM, policy and socioeconomic research, and enhancing institutions. They may be classified by commodities, agroecologies, themes as well as scientific disciplines.

The organization of research in the CGIAR has gone through a transition over the past decade. For the purpose of defining programme structure, accounting and governance, the System's totality of research and research related activities are organized and implemented,

\footnotetext{
${ }^{5}$ While this is not a final and definitive outline of programme organization, it is necessary to lay out the broad programme thrust in order to be more specific on assessing relevance and quality of research.
} 
accounted for and governed as a continuum of three basic types or categories of programmatic arrangements, namely: (i) Centre Core Programmes ${ }^{6}$; (ii) Systemwide Programmes $(S W P s)^{7}$; and (iii) Challenge Programmes (CPs). ${ }^{8}$ (Figure 1).

\section{The Programmatic Context}

The distinguishing characteristics of the three major forms of programmatic organization of research are outlined herebelow.

Centre Core Programmes: The System's research and research-related activities are basically organised around the autonomous international Centres. The activities are planned, implemented, monitored and evaluated, and accounted for by the Boards and managers of the individual Centres/Institutes. The activities are supported by the Group members and other donors by way of unrestricted core funds and as restricted core and/or project support. Many contributions are also received in kind i.e. through secondment of scientific staff, equipment donations, as well as use of facilitation and experiment stations.

In addition to those activities directly and exclusively conducted by the staff of the individual Centres, are many research and research-related activities implemented and jointly conducted by the Centres with national and other partner institutions through various networks and consortium which the Centres have organised and led.

These two sets of activities constitute the Core Programme of an individual Centre, all of which are accounted and reported under the supervision and control of the Centre Board. The Centre Board in turn is accountable for their activities to the Group and other donors.

Systemwide Programmes: Systemwide Programmes cover sets of activities conducted by the Centre among themselves and with various national and partner institutions with specific objectives in mind. However for the most part they are organised and recognised as such to provide scientific coherence to the totality of System activities on a specific theme or problem area and to promote efficiency and effectiveness in their planning, implementation and delivery. They are usually activities no individual Centre can pursue on its own with equal success and those activities which clearly are best conducted in partnership with others.

To provide coherence, direction and scientific quality control on these activities, a scientific Steering Committee is organised by mutual consent among the partners. The Steering Committee is composed of key scientific investigators representing the partner institutions, among whom one is designated as Systemwide Programme leader/co-ordinator, director or appropriate.

\footnotetext{
${ }^{6}$ Research in the Centre Core Programme is supported through unrestricted and restricted donor funding.

${ }^{7}$ Include global and regional subject matter or thematic programmes, and programmes with an ecoregional approach.

${ }^{8}$ Challenge Programmes are the latest modality adopted by the CGIAR System. These projects generally are large, focused on solving a given major issue in a finite, defined time period, represent partnerships with nonCGIAR organizations, and involve cross disciplinary activity.
} 
In recognition of initiative, scientific leadership and/or substantive resource commitments, the co-operating partner turn to one of the Centres to become lead or convenor, and to reduce transaction costs as well, the finance and administration of the Systemwide Programme are entrusted to the Board and management of the lead/convenor Centre.

The designated Systemwide Programme leader is usually a key scientific leader/manager of the convenor Centre and is endowed by the convenor Centre and the member parties with appropriate management and decision-making authorities.

A key feature therefore of Systemwide Programmes is that governance and accountability to the Group, as well as to the co-operating partner institutions, are entrusted to the management and Board of the convenor Centre.

Challenge Programmes: Challenge Programmes are organisational instruments designed not only to provide coherence and direction to System activities on a specific theme or problem area and to promote efficiency and effectiveness in their conduct. They are likewise intended to provide the System's impact on its stated goals of poverty alleviation, promoting food security and enhancing the sustainability of natural resources by drawing in new, additional partners particularly those involved in development and utilisation of the System's research outputs, as well as those institutions leading global efforts in conventions and initiatives with similar and/or related goals.

By articulating the System's activities to those global activities related to poverty alleviation, food security and environment protection by way of Challenge Programmes, the System's activities hopefully will be given the attention and political support they deserve.

As in Systemwide Programmes, a scientific Steering Committee will normally provide coherence, direction and science quality control over the activities of each Challenge Programme. However in view of the significant involvement and commitments of partner institutions beyond the CGIAR Centres themselves and their regular NARS counterparts, a Stakeholder Board may usually be necessary to provide accountability and formal governance to these global partnerships or alliances. The Stakeholder Board of a Challenge Programme will consist of individuals representing the perspectives/interests of its manifest stakeholder.

The terms of reference, composition, costs and details of these administrative, management and governance arrangements and structures will be as provided in the Challenge Programme's business plan agreed to by the partner, and approved by the CGIAR.

\section{Role of the Science Council \\ Challenge Programmes are chosen through a competitive process that involves the SC. The SC provides the process for assessing, weighing and developing recommendations for choice of CPs; it forwards a list of recommended priority CPs to the Executive Council, which then decides on which CPs are to be funded and at what levels. In the process of assessing CPs, the SC also should take on the role of recommending improvements to the organizers of such.}


In the category of Systemwide Programmes, CGIAR currently has eighteen Programmes ongoing in various stages of implementation, of which eight are classified as ecoregionally focused SWPs. Decisions remain to be made by the Executive Council on the way forward in terms of organizing new SWPs. The iSC currently is in the process of debating its perspective on SWP modalities and on the most desirable and effective means of proceeding with consideration of new SWPs, of which several ideas already are on the table, with some already ongoing as inter-Centre activities.

With regard to the organization and balance of Centre core programmes, the role of the SC is to evaluate and comment on Centre medium term plans (MTPs). The SC forwards the results of such deliberations to the Executive Council for use in making its decisions on the level of investment in the CGIAR. In the process of the SC's deliberations it could provide useful input for future planning in the Centres. It may be desirable to develop a more operational and systematic mechanism for assuring such feed back to Centres.

\section{IMPLEMENTING, MONITORING, AND EVALUATING RESEARCH IN THE CGIAR SYSTEM}

Research in the CGIAR is undertaken by the Centres with their partners through Core Programmes, Systemwide Programmes, and, in the future, through Challenge Programmes with outside partners. In fact, implementation of most CGIAR research involves partners, most often right from the initiation of the research planning leading up to the research. As such, mechanisms need to be developed to include such partners in the monitoring and evaluation of research. That implies development of sound accountability mechanisms, where such can be shared appropriately among partners.

The Centres and their Boards ultimately are responsible for monitoring and evaluating their own research for both quality and relevance. The System, primarily through its SC, monitors and evaluates the adequacy of such Centre monitoring and evaluation activities to be able to ensure the members of the Group that the Centres are producing transparent, quality results in their internally mounted evaluations. On occasion, the SC would mount its own, independent evaluation of the Centre's science relevance and quality.

In addition to Centres, the System has SWPs and CPs. M\&E for these cross-Centre Programmes could be undertaken through the Programmes themselves, and through independent evaluation mounted by the SC.

\section{Guiding Principles}

Any scientific endeavour that aims at being relevant and of high quality should be both effective and efficient in its conduct. In most, if not all, scientific institutions, there exist mechanisms to ensure that research programmes are carried out as effectively and efficiently as possible, as part of the process of self-accountability as well as of accounting to Investors 
for resources utilized in research. Such mechanisms are based on both internal or self and external evaluation ${ }^{9}$ processes.

Self-evaluation and assessment is an integral part of responsible management. Performing institutions invariably have quality control mechanisms in place to set benchmarks or standards as well as for monitoring progress (or lack of) towards meeting the agreed standards.

In the CGIAR Centres, this function of assurance of relevance and quality of science is implemented through:

- Self-assessments and internally managed external or peer reviews as an integral part of responsible management and governance;

- Built-in mechanisms for supervision and personnel evaluations by the Centre managements and the Boards;

- Recruitment and retention of world class scientists, including attractive conditions of service;

- Supervision exercised through research programme leaders, research directors, DDGResearch;

- Policy oversight by Programme Committees of the Boards which are guided by the longer-term strategic plans and medium-term operational plans;

- Ex-ante reviews of projects and programmes by programme leaders and Directors of Research, sometimes with external peer input; and the various internally managed review and evaluation processes, including peer reviews of particular projects and activities, peer participation in annual programme reviews.

- Ex-post reviews which have included the strengthening of impact assessment in recent years.

While self-assessment processes involving internal experts is a necessary element, Centres increasingly recognize the value of inviting external experts and peers in assisting to enhance relevance and quality of science. In the same vein, there is now a better appreciation of the pivotal role of the Centre Boards and management on quality assurance. However, there is still important room and need to strengthen ex-ante science review processes in the Centres, on a project by project basis. Further, given the need for incorporating greater concern for higher standards of quality in the CGIAR science culture, the achievement of highest scientific relevance and frontier level quality must become more than ever before the

\footnotetext{
${ }^{9}$ The word evaluation is an abstract noun, and can mean different things to different people unless it is defined explicitly. The iSC defines evaluation as follows. Evaluation is to do with finding or ascertaining or appraising or assessing the value of an activity, event, object, etc. The word value refers to the worth, desirability, utility and the qualities on which these depend. Thus, in the context of science relevance and quality, evaluation is defined as a process and products thereof undertaken to examine the value in terms of relevance and quality of past, present or proposed activities in relation to their purpose and context. Evaluation of a proposed activity or theme is referred to as ex-ante evaluation, and so are evaluations (perspective studies, strategic studies) undertaken for planning or setting planning context and formulating priorities and strategies; evaluation undertaken during the implementation of an activity is referred to as performance monitoring or monitoring and evaluation; and evaluation undertaken on a completed or a past activity is referred to as ex-post evaluation which may also include ex-post impact assessment.
} 
personal cause and the guiding value for each researcher in the System, for each research manager, for each Centre Director and administrator.

Donors also undertake evaluation specially for restricted research projects, but also when they provide fully or targeted unrestricted support in which case broad strategies and outputs and outcomes are examined.

However, for scientific research, the necessary internal assessments, however effective, are not deemed sufficient. Independent external evaluations by outside peers, both ex-ante and ex-post are de rigeur to enrich approaches, to enhance scientific credibility as well as to foster accountability to investors for resources expended in the enterprise. While some progress has been made recently in promoting ex-post peer assessments, systematic mechanisms for ex-ante peer evaluation processes have yet to be introduced in the CGIAR System.

Thus, the relevance and quality of the research practised in the CGIAR should continue to be assessed through both internal and external evaluation processes. There is a need to build a genuine evaluation and impact culture in the System, including amongst Investors, within the SC, the Centres and with each scientist in the Centres. Within such a culture, a much clearer focus on outputs and impacts within the Centres and in other entities within the System is highly desirable.

The key to independence in assessment is that it be transparent, objective, and unbiased in terms of selection of subject (programmes and projects) and in process. Consequently, even when major proportions of the Centres' portfolios are made up of special projects reviewed directly by investors, there was a perceived need for independent evaluation by external peer sources that do not have a vested interest in the activity being evaluated.

While accountability assurance is a main purpose for much of the evaluation in the System, an equally important and closely linked purpose is to provide information and feedback for the strategic planning of new areas of emphasis and areas to diminish or phase out. It is evident that such planning should draw heavily on the self-evaluations and impact assessments carried out in the Centres and within the context of the regional fora and other groups focusing on regional research needs and priorities. Also, in shifting the focus towards CGIAR outputs and impacts, special care needs to be taken to ensure balance between immediate or short term outputs/impacts and the quality of science and research that will help ensure the flow of future outputs and impacts.

\section{Internal Centre Evaluation Processes}

At the Centre level, the internal evaluation process would proceed as follows:

With oversight from the BOT, Centre management will design and implement a comprehensive continuous self-evaluation procedure. It will cover the relevance of research and related activities, including planning for relevance and stakeholder consultations; the quality of scientific inputs and research methodologies, outputs, intermediate outcomes and impacts; and the efficiency and effectiveness of operations, including research partnerships 
and delivery of research products. Centres should develop procedures for in-house peer assessments of research proposals both ex-ante and ex-post, making use of the existing expertise in the network of CGIAR Centres, as well as procedures for the review of specific areas and programmes. To the extent necessary, keeping in mind the costs involved, the Centres are encouraged to engage external expert reviewers in these processes.

At the programme level, there should be mechanisms to operate the internal monitoring and review of planning processes, achievement of milestones, roles of partnerships and all other specific activities. The template for these has been installed in the log-frame planning format. In all cases, the output from the internal evaluation processes are expected to provide an input into the external independent processes overseen by the SC which also assesses the appropriateness and effectiveness of the internal evaluation processes for assurance of science relevance and quality.

It is extremely important to set out clearly the positive image of ex-ante peer evaluation as an instrument for enriching each research proposal before it is approved for funding, with benefits of knowledge and creative suggestions from groups of researchers invited to join in the peer evaluation process. The author of each proposed research project will gain from the state of the art knowledge on the subject and from additional suggestions from peers regarding investigation techniques, design of experiments, consultation with beneficiaries, etc. This should counter in a positive and constructive way the fear about exante peer evaluation as a simple administrative or "police" procedure. Peer evaluation can also sharpen the relevance of science, not only its quality, and could definitely help promote the best use of financial resources in the service of the most relevant and high quality research proposal. Further, ex-ante peer evaluations will facilitate bringing into the CGIAR System ideas, knowledge, and techniques from outside the System by inviting outside researchers to mix with in-house researchers in the peer evaluation rounds. In short, to use the words employed during the AGM2001, "we need a world class peer review system to help produce world class quality research."

Consistent with the guidance given at AGM 2001, the iSC has initiated the preparation of guidelines for introducing state of the art mechanisms for ex-ante peer review of proposed research projects, or for strengthening it where it already exists. The AGM emphasized that ex-ante peer review should be applied to all research projects, rather than only to the new CPs. The iSC is in the process of commissioning a study on existing good experiences with ex-ante review mechanisms outside and inside the CGIAR. On the basis of the results of this study, the SC would prepare recommendations that would provide guidelines for the design of an ex-ante peer review process, tailored to the objectives of enhancing the quality and relevance of research and science in the CGIAR, and periodically evaluate its effectiveness. The proposition is that Centres will design and implement a self-evaluation procedure. It seems that more emphasis needs to be placed on the role of and procedures for peer reviews, with guarantees of anonymity for peer reviewers as a routine procedure for at least a significant share of the review exercises (clearly not for all when reviewers are teams of experts coming to a Centre). Centre research plans and scientific papers can be submitted for external peer reviews through anonymous procedures. DGs would be expected to have 
consulted in this fashion the most respected experts in the field. Anonymity and freedom to criticize are essential to peer reviews in advanced research institutions.

While the Boards' oversight role of the internal evaluation processes has improved in recent years, there is much more potential for incisive and in-depth engagement of Boards in quality assurance processes beyond their traditional role of monitoring and approving overall programmes and LTPs and MTPs. There is enormous scientific competence embodied in the memberships of Centre Boards and Board members should be used more intensively for quality enhancement purposes. For instance, working groups could be constituted among members of each Board requiring them to participate in the ex-ante peer evaluations of individual projects.

The Centres are long standing, well organized institutions that are well-placed to develop, implement and maintain the self-assessment mechanisms described above on a continuing basis. Basically, the proposal is to rely much more on a Centre managed external peer review system (PRS). This approach is put forth on the premise that Centre BOT processes and performance are assured. With such assurance (which can be monitored in several ways), preliminary thinking is that the rest of the centre review process might proceed as follows:

- With oversight from the BOT, Centre management will design and implement a continuous self-evaluation procedure that focuses on efficiency in operation, quality of science outputs and relevance of impacts. The core of this approach will be the PRS mentioned above. The SC will prepare guidelines for consideration by centres in preparing the procedures.

- The overall self-evaluation process would include, at a minimum, procedures for evaluating:

o the relevance and impact of the research, both on-going and completed;

$\circ$ the quality of the science used

$\circ$ the efficiency with which research resources are used (including an assessment of the appropriate role of partnerships and capacity strengthening activities in meeting the centre's goals).

- The evaluation should focus on both present outputs and impacts and on the quality and relevance of ongoing science as the most objective indicator of potential impact. This should include an assessment of the quality of staff, recognising that good science is only possible with good staff.

- Results of the self-evaluations would be adapted to the common format provided by the Logframe, currently adopted by all Centres. This information will be used to consolidate the outputs, milestones and impacts of each project into a central Management Information System (MIS) for the whole CGIAR, including the individual centres.

- This information would be systematically reviewed (annually) by SC in its oversight capacity. SC would report the results of its review in an annual "Science Report" to the Group and executive body of the CGIAR. This would emphasize scientific 
breakthroughs and notable achievements, as well as gaps and constraints. This would serve as a benchmark against other relevant scientific institutions and help document science progress over the CGIAR System as a whole.

It is important to recognise that the development of a centre focused self-evaluation process would take time. Although this would be an evolutionary and learning process, the steps of transition must be planned carefully. The SC would be expected to prepare new programme evaluation and assessment guidelines and benchmarks in consultation with centres, investors and other interested stakeholders of the System and develop an appropriate management information system as the centres gear up for a full scale internal-review process.

\section{External Independent Evaluation Processes}

External independent ${ }^{10}$ assessment and advice has always been a key pillar in guarding and enhancing the quality and relevance of the CGIAR's research. From its inception, a Technical Advisory Committee (TAC) composed of knowledgeable scientists representing a broad range of disciplines drawn from all over the world was organized to provide the Members of the Group as well as the Centres independent, objective and rigorous advice on research objectives, directions, priorities and strategies as well as assurance of relevance and quality of science practised at the Centres.

Under the SC, external evaluation at the Centre level would be of two kinds:

Periodically (roughly every five years), an independent external programme (or science) evaluation of each Centre will be commissioned by the Science Council ${ }^{11}$. The evaluation will be organized and managed by the Science Council with the help of its Secretariat. The evaluation will concentrate on the Centre's processes for assessing the relevance, quality and impact of research and related activities, and on the efficiency of resource use. The evaluation will also help the Centre reassess its strategic vision and set new research priorities. The evaluation will draw heavily on the results of the internal evaluations and assessments, and thus will supplement the Centre-managed process. It may thus be considered to be an 'audit of audit processes' and will be far less time consuming than the current external programme and management reviews.

The second kind of external evaluation at the Centre level should address the mediumterm plans (MTPs). Centre MTPs will be assessed as a group every 3 years by the SC, to provide the CGIAR with a regular, complete overview of the System's research agenda and apparent gaps, and an evaluation of the overall agenda in the context of the CGIAR's mission and goals. Information from the external evaluations will feed into this process. The MTPs will then constitute the basic documents against which Centre performance will be evaluated.

\footnotetext{
${ }^{10}$ The word independent here refers to the basic purpose of the SC which is to ensure that its advice to the Group and CGIAR System is objective and impartial in addition to being transparent in terms of the advice is formed or developed.

${ }^{11}$ When the SC commissions an independent evaluation, it does so on behalf of the Group, deploying expert panels or individual experts depending on the task at hand. The experts serve in a personal capacity.
} 
This implies a need to more precisely define and articulate System priorities across sectors, commodities, themes and logframe outputs. In this context, Centre MTPs can be more objectively and systematically analysed.

At the SWP and CP levels, the SC would commission external science evaluations at the various stages of Programme development and implementation in the context of System level resource allocation to geographical regions, sectors, commodities, Centres, Programmes, enterprizes and outputs. (It should be noted that SWPs and, particularly the more independent CPs also should have well developed and articulated internal monitoring and evaluation processes).

\section{Evaluation of Systemwide Programmes and Challenge Programmes}

The iSC has had to develop initial criteria and procedures for evaluation of concept notes and pre-proposals on the new CPs. Further analyses and refinements of such criteria are being made as well as for the guidelines and criteria for evaluation of the final CP proposals. Similar approaches will be proposed for the evaluation of Systemwide programmes based on the revision, in consultation with $\mathrm{CBC}, \mathrm{CDC}$ and other stakeholders, of the current TAC/iSC document on the external evaluation process for Systemwide Programmes.

A several step process has been put in place to review and assess Challenge Programmes. This allows Centres and their partners to progressively invest in the programme as their probability of CGIAR approval and support grows. The role of the iSC is to bring the best scientific judgement to bear, through internal iSC expertise and through panels of external reviewers who collectively have knowledge of the relevant cutting edge science and of its application to development needs. Those panels include scientists and development specialists from advanced laboratories in both the north and the south, and representatives from both developing country NARS and NGOs. The external review process is broadly selective, and protects the anonymity of the reviewers according to procedures widely used in proposal review in other institutions.

In the pilot CP phase priority was given in the concept note and pre-proposal phases to the scientific potential for problem solution given the predominant capability and comparative advantage of the Centres, and the potential for major impact on poor people and on critical and growing environmental problems. Evaluations were made on strategic issues and very general statements about scientific approaches. In the review of full proposals the programmes will become much more specific. The role of the iSC now turns to assisting the proponents of the three selected programmes to formulate them in the most effective way through feedback from external reviewers and from the iSC commentary.

It is envisioned that the CPs will go through an evolutionary process as governance structures are put in place, partnerships consolidated and work plans evolved. It is assumed and strongly recommended that all CPs and SWPs be immediately brought into the MTP process following the CGIAR logical framework structure. As TAC conducted its overall review of SWPs with an ecoregional approach and more recently of theme-specific SWPs, a major constraint in the review process was the inability to sort out normal Centre Core programme activities from those of SWPs. If such SWPs are to be held accountable they must 
have identifiable structure. The structure must be consistent with the logframe process of the CGIAR. That logframe was developed, in fact, anticipating the complex processes of future programmes.

The Science Council should carefully review the first MTPs (business plans) of the CPs to assure that the various elements of the logframe are in place: Beneficiaries, goals, objectives, intermediate goals and milestones should be clearly identified. Impact pathways should be clear. Programme evaluation and impact assessment would then follow internal and external review processes similar, but not necessarily identical to other Centre and programme reviews. The System should bring all Systemwide Programmes under this same logframe-based MTP and programmes review process.

The financial structure will be complex for partnerships formed in the new CPs, with combinations of targeted and attributed funding. This structure should be clear, with the ExCo and System Office overseeing its clarity. The problem of redundancy or overlaps in accounting also needs to be clarified. As subsidiarity is a key element and a fundamental principle of all of these programmes, the resources will appear in more than one place and must be sorted out in aggregation at various levels.

Scientific accountability will not necessarily (and probably never will) coincide in scope, with financial accountability given the attributed nature of many of the resources brought by non-CGIAR partners who are not operating under contract. The attributed resources should be footnoted only according to standard accounting procedures within the CGIAR System, but the scientific contributions of such partners may be critical to the success of the project. In programme evaluation the review process must then assess the adequacy of output of those partners, even though they are not strictly accountable in a financial way. This has to be done with discretion and appropriate sensitivity, but it is critical to programme success and accountability. Scientific accountability and review will almost always be more broad than financial accountability. Review panels and all partners should understand this distinction.

Once the pilot $\mathrm{CP}$ process is complete and the second round is more advanced there is an urgent need to reassess the role of Systemwide Programmes in relation to CPs and centre core programmes. There seems to be a need for such "intermediate structure" programmes with less formal governance to tackle more specific issues. The "why and how" for these SWPs should be articulated with respect to CPs. That will be an issue for the regular CP process which is now underway. The new SC will have a central role to play in this process.

Based on the AGM2001 interpretation of the new responsibilities (Annexes 1 and 2), the SC is expected to undertake periodic science evaluations of individual Centre project portfolio when Centres produce fresh MTPs every 3 years, and of the total project portfolio of the CGIAR System, referred to as the CGIAR research agenda. Also, the SC is expected to undertake ex-ante evaluation of Challenge Programmes, and monitor and evaluate their performance during implementation in terms of science relevance and quality. The focus on outputs and impact also requires that emphasis is placed in the SC to carry out impact assessment studies in a systematic fashion. This is discussed in the next section. 


\section{IMPACTING POVERTY, ENVIRONMENT AND FOOD SECURITY}

Ultimately, the objective and purpose of CGIAR research and related activities is to have positive impact on reducing poverty, enhancing the environment and its sustainable use, and food security. This impact focus has to be built into every CGIAR project, whether at the Centre or cross-Centre and Challenge Programme levels.

\section{Impact Culture Context}

Centres are in varying stages of introducing an "impact culture" into their daily operations. Most Centres undertake impact assessments, again with varying levels of sophistication and success. A great deal of discussion and activity in this area is on going in the System, and more is needed in the future, as the need for increasing efficiency and effectiveness in programme design, development, implementation increase. Centres are increasing being held tightly accountable for their use of resources. At the same time, there is increased need for assessments of impacts to inform internal planning of programmes and activities and in setting priorities among alternative uses of resources. All units making up the CGIAR have a role to play in furthering the effectiveness of impact assessment in the System.

\section{Role of the Science Council}

How impact assessment (IA) activities are organized in the $\mathrm{SC}$ remain to be determined. However, the SC should ensure that the strong operational linkages to the forward planning (see section 2) and monitoring and evaluation of on-going programmes (see section 4) be maintained. The three functions are complementary and depend on each other in a dynamic feedback process that is so essential to assuring that the CGIAR remains on the frontiers of science and development, maintains its science quality and relevance, and contributes in the most effective and efficient manner to the goals of the Group.

Within this broader context of CGIAR forward planning, monitoring and evaluation of on-going programmes, and accountability to investors for past use of their resources, the System needs:

(a) evidence of the impacts of its various completed and on-going research and related activities,

(b) impact information useful in understanding appropriate and desirable changes in direction of the System's programmes, and

(c) mechanisms for strengthening the capacity to do impact assessment in the System's Centres.

Within this context, the SC IA activity should:

- work with Centres in strengthening Centre IA capacity and linking activities;

- undertake System level impact assessments that involve inter-Centre cooperation and collaboration;

- work more closely with the System's science monitoring and evaluation activities to ensure that the complementarities between IA and M\&E are fully realized; 
- provide ex-post impact assessment outputs that can serve the purposes of planning the new Challenge Programmes and reorienting overall System plans, i.e., work more closely with the forward looking planning activities performed at System level;

- open up more broadly to the outside evaluation/impact assessment world, including through networking, outsourcing and implementing some of its future studies on a broader, competitive "request for proposal" basis, and

- develop a systematic process to assure high quality, independent scientific peer review of all its analytical studies and their results.

Specifically, there is a continuing need for four main ex post impact assessment functions at the System level within the CGIAR:

(1) Conducting high quality, independent impact assessments to provide results of use to (a) investors, in justifying their investments; and (b) System management and Centres in planning their programmes and investments and developing and allocating budgets.

(2) Tracking information related to the impacts associated with Centre and cross Centre activities. This could involve routine data collection; and developing, maintaining and managing, in collaboration with the planning and monitoring and evaluation units, an appropriate data base/MIS for the System that would provide annual updates on accomplishments (training, research, etc.) in addition to data on other indicators of relevance in understanding the impacts of the System's outputs and processes.

(3) Developing methodologies, providing training in their use, and providing advice and facilitation for Centres as needed, e.g., in terms of setting up programmes and projects in such a way as to make tracking and analyzing impacts more feasible, transparent and of high quality. ${ }^{12}$ This would include the "certification" of quality of internal IAs and organizing and "certifying" quality of external IAs. Ideally, this function would involve the establishment and maintenance of a CGIAR wide IA web site that also would be open to all outside entities with an interest and involvement in IA related to agricultural research and training.

(4) Delivering and facilitating the most effective use of the outputs of the IA entity, e.g., facilitating Centre interaction and learning, and development an effective impact culture in the Centres. Centre scientists have adapted rapidly to the output-orientation of the Systemwide logframe, but in the more complex projects that they are working in, the connectivity of their outputs to impact may not always be evident. Quantification through impact assessment greatly helps in underscoring an impact-oriented culture. It also would involve providing insights to investors on what is and is not feasible in terms of carrying out IAs for such activities as natural resources management, social science research and capacity strengthening.

\footnotetext{
12 It should be stressed that the implication of this statement is not that the Centres are lacking in high quality impact assessment capacity. Rather, the thinking here is that a central entity can facilitate interaction among Centres, gain access with System level resources to expertise needed by all Centres, and provide a clearing house for information and documentation of use to all Centres. In a sense, this central entity will provide "System level public goods."
} 
Impact assessment is a central function of the $\mathrm{SC}$, in agreement with the recommendations coming out of AGM01. The synergies between impact assessment and the overall mandate to guard the quality and relevance of science are strong.

\section{LINKING THE PAST AND THE PRESENT WITH THE FUTURE: THE FEED BACK DYNAMICS}

One of the strengths of the CGIAR has been the existence of feed back mechanisms where Centres and the System learn from the past mistakes and successes and adjust programmes and organizational mechanisms to the lessons learned.

\section{Context}

The CGIAR exists in a dynamic environment of evolving science and changes in development that should influence the priorities and activities of the CGIAR. Mechanisms need to be developed to make sure that relevant lessons from the past and present feed into plans for the future of the System. This is a major part of guarding and enhancing science quality and relevance.

\section{Role of the Science Council}

The SC should build effective working links between its functions of impact assessment - learning about past successes and failures in terms of moving towards CGIAR goals, performance monitoring or monitoring and evaluation - learning about on-going programmes and the state of implementation of current plans, and forward strategic planning - bringing together the best available knowledge of outputs, impacts, relationships, trends, and changes in the scientific and development environments to develop plans for future priorities and strategies of the CGIAR. Such linkages can be developed by establishing working relationships between those in the SC and its Secretariat who become focal points for its forward planning, monitoring and evaluation, and impact assessment activities. The results of activity coming out of such working relationships can help generate more effective and efficient programmes in the System, including better quality and more relevant science. The SC should explicitly build into its programme mechanisms to ensure that the particular dynamics of the CGIAR System is fully exploited in moving ahead.

Acknowledgements. The authors would like to thank the members and staff of the interim Science Council for their input, and the two anonymous referees for valuable comments on the manuscript.

\section{REFERENCE}

TAC (2000). A Food Secure World for All: Towards a New Vision and Strategy for the CGIAR. Technical Advisory Committee, FAO, Rome. 
Figure 1: Organizational Design of the CGIAR

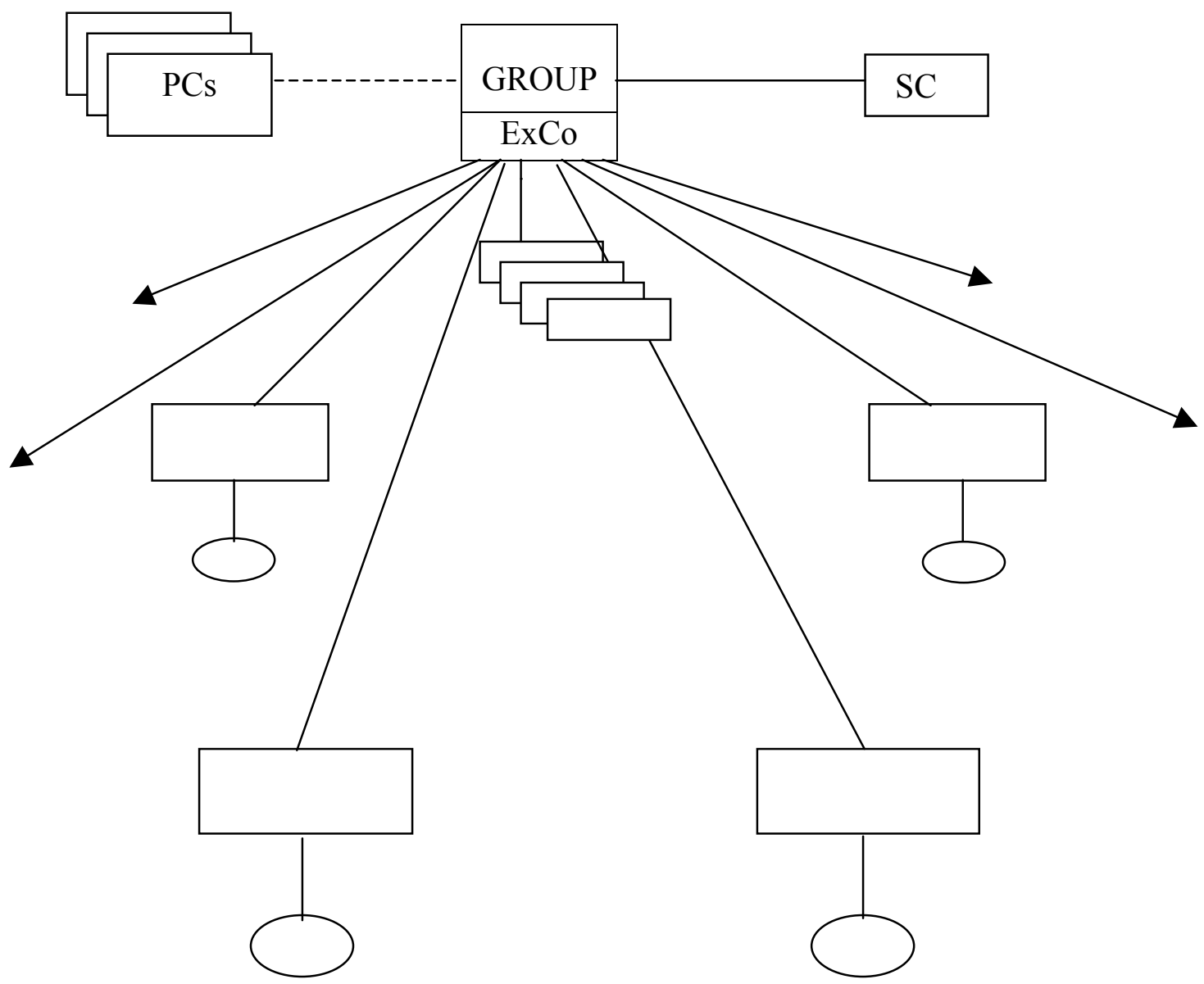

$\square \quad$ Inner core of Centres (16)

$\longrightarrow$ Outer Network of Challenge Programmes

$\infty$

$\longrightarrow$

Formal reporting responsibilities

Co-ordinating, advising relationships

(Centres, CPs and SWPs are interconnected

by multiple dotted lines, not shown in order not to clutter diagram)

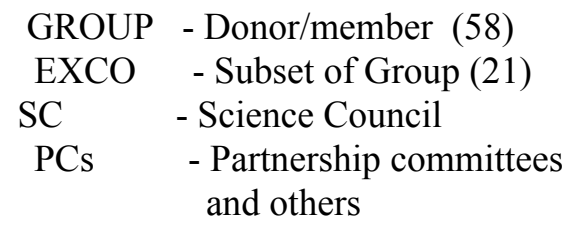


Table 1: Overview of Suggested Functions of the Science Council in the overall Context of CGIAR Activity and Organization

\begin{tabular}{|c|c|c|c|}
\hline CGIAR Processes & Context & $\begin{array}{l}\text { Role of CG Entities other than } \\
\text { the SC }\end{array}$ & Complementary Role of SC \\
\hline $\begin{array}{l}\text { Research planning and } \\
\text { strategy development }\end{array}$ & $\begin{array}{l}\text { Outcomes should depend on: } \\
>\text { external science and } \\
\text { development environment } \\
>\text { comparative advantage }\end{array}$ & $\begin{array}{l}\text { ExCo decision at System level and } \\
\text { centres at centre level }\end{array}$ & $\begin{array}{l}\text { SC provides input and advises on } \\
\text { appropriate strategy and overall } \\
\text { System planning to assure quality } \\
\text { and relevance of research; }\end{array}$ \\
\hline Organizing research & $\begin{array}{l}\text { CGIAR research organized in Centre } \\
\text { Core Programmes, inter-Centre } \\
\text { Systemwide Programmes and } \\
\text { Challenge Programmes working } \\
\text { closely with outside partners }\end{array}$ & $\begin{array}{l}\text { Centres sort out their mix of } \\
\text { involvement with different } \\
\text { organizing mechanisms }\end{array}$ & $\begin{array}{l}\text { SC is centrally involved in } \\
\text { competitive process for CPs } \\
\text { and in organizing and } \\
\text { shaping the SWPs; } \\
\text { SC provides advice on centre } \\
\text { MTPs and other plans; }\end{array}$ \\
\hline $\begin{array}{l}\text { Implementing, monitoring } \\
\text { and evaluating research } \\
\text { (incl. Management and } \\
\text { governance) }\end{array}$ & $\begin{array}{l}\text { Centres face budget and resource } \\
\text { issues; partnership issues, etc. }\end{array}$ & $\begin{array}{l}>\text { Primarily centre function to } \\
\text { produce outputs; } \\
>\quad \text { Centres perform internal } \\
\text { M\&E, incl.peer reviews; } \\
>\text { CCERs; } \\
>\quad \text { ExCo through SO monitors } \\
\text { and evaluates management } \\
\text { and governance, including } \\
\text { board performance }\end{array}$ & $\begin{array}{l}>\text { SC manages and provides } \\
\text { oversight to M\&E process to } \\
\text { assure quality and relevance } \\
\text { of science; } \\
>\quad \text { SC performs external } \\
\text { reviews as needed; } \\
>\text { SC supports Systemwide } \\
\text { data base on centre and } \\
\text { System outputs, } \\
\text { performance, etc. }\end{array}$ \\
\hline $\begin{array}{l}\text { Impacting poverty, } \\
\text { environment and food } \\
\text { security; }\end{array}$ & $\begin{array}{l}\text { Where CGIAR activity becomes } \\
\text { relevant to its goals }\end{array}$ & $\begin{array}{l}>\text { Centres measure impact of } \\
\text { their activities; } \\
>\text { ExCo monitors centre } \\
\text { impacts for CGIAR } \\
\text { members }\end{array}$ & $\begin{array}{l}\text { SC performs impact } \\
\text { assessment at System level; } \\
\text { SC supports Systemwide } \\
\text { data base and information } \\
\text { centre for impacts; }\end{array}$ \\
\hline $\begin{array}{l}\text { Feedback into planning } \\
\text { and strategy }\end{array}$ & $\begin{array}{l}\text { Creating the dynamics of the CGIAR } \\
\text { System; learning by doing }\end{array}$ & $\begin{array}{l}\text { Centres do this at Centre level, } \\
\text { feeding information to System; }\end{array}$ & $\begin{array}{l}\text { SC does this through its continuing } \\
\text { planning processes }\end{array}$ \\
\hline
\end{tabular}

